\title{
A PALEY-WIENER THEOREM FOR BERGMAN SPACES WITH APPLICATION TO INVARIANT SUBSPACES*
}

\author{
Peter Duren, Eva A. Gallardo-Gutiérrez, \\ And Alfonso Montes-Rodríguez
}

Abstract. An analogue of the Paley-Wiener theorem is developed for weighted Bergman spaces of analytic functions in the upper half-plane. The result is applied to show that the invariant subspaces of the shift operator on the standard Bergman space of the unit disk can be identified with those of a convolution Volterra operator on the space $L^{2}\left(\mathbb{R}^{+},(1 / t) d t\right)$.

\section{INTRODUCTION}

Let $\Pi^{+}$denote the upper half of the complex plane. Recall that the Hardy space $\mathcal{H}^{2}\left(\Pi^{+}\right)$consists of the functions $F$ analytic on $\Pi^{+}$with finite norm

$$
\|F\|_{\mathcal{H}^{2}\left(\Pi^{+}\right)}=\left\{\sup _{0<y<\infty} \int_{-\infty}^{\infty}|F(x+i y)|^{2} d x\right\}^{1 / 2}
$$

A classical theorem of Paley and Wiener ([6]; see [8] or [2]) states that $\mathcal{H}^{2}\left(\Pi^{+}\right)$ is isometrically isomorphic under the Fourier transform to $L^{2}\left(\mathbb{R}^{+}\right)$, the space of functions square-integrable over the positive real line. In fact, to each function $F \in \mathcal{H}^{2}\left(\Pi^{+}\right)$there corresponds a function $f \in L^{2}\left(\mathbb{R}^{+}\right)$such that

$$
F(z)=\int_{0}^{\infty} f(t) e^{i z t} d t, \quad z \in \Pi^{+}
$$

and

$$
\|F\|_{\mathcal{H}^{2}\left(\Pi^{+}\right)}^{2}=2 \pi \int_{0}^{\infty}|f(t)|^{2} d t .
$$

Moreover, Plancherel's theorem asserts that the Fourier transform

$$
\widehat{F}(t)=\frac{1}{2 \pi} \int_{-\infty}^{\infty} F(x) e^{-i t x} d x, \quad t \in \mathbb{R}
$$

coincides with $f$. In particular, $\widehat{F}(t)=0$ for almost every $t<0$.

1991 Mathematics Subject Classification. Primary 30H05; Secondary 46E20, 47A15.

Key words and phrases. Bergman spaces, Paley-Wiener theorem, Fourier transform, Laguerre polynomials, invariant subspaces, Bergman shift, convolution operators, Volterra operators.

Partially supported by Plan Nacional I+D grant no. BFM2003-00034, and Junta de Andalucía FQM-260. Second author also supported by a grant from Secretaría de Estado de Educación y Universidades, Ref. PR2004-0584.

* Accepted for publication in Bulletin of London Mathematical Society 
We begin this note with an analogue of the Paley-Wiener theorem for Bergman spaces, which will be applied to the shift operator on the standard Bergman space $\mathcal{A}^{2}(\mathbb{D})$ over the unit disk $\mathbb{D}$. Before stating the results, we need to introduce some terminology.

For $\alpha>-1$, the weighted Bergman space $\mathcal{A}_{\alpha}^{2}\left(\Pi^{+}\right)$on the upper half plane consists of those functions $F$ analytic in $\Pi^{+}$for which

$$
\|F\|_{\mathcal{A}_{\alpha}^{2}\left(\Pi^{+}\right)}^{2}=\int_{\Pi^{+}}|F(x+i y)|^{2} y^{\alpha} d x d y<\infty .
$$

For $\beta>0$, let $L_{\beta}^{2}\left(\mathbb{R}^{+}\right)$denote the space of complex-valued measurable functions $f$ on $\mathbb{R}^{+}$for which

$$
\|f\|_{L_{\beta}^{2}\left(\mathbb{R}^{+}\right)}^{2}=\frac{2 \pi \Gamma(\beta)}{2^{\beta}} \int_{0}^{\infty}|f(t)|^{2} t^{-\beta} d t<\infty .
$$

We can now state the Bergman-space analogue of the Paley-Wiener theorem.

Theorem 1. For each $\alpha>-1$, the space $\mathcal{A}_{\alpha}^{2}\left(\Pi^{+}\right)$is isometrically isomorphic under the Fourier transform to the space $L_{\alpha+1}^{2}\left(\mathbb{R}^{+}\right)$. More precisely, $F \in \mathcal{A}_{\alpha}^{2}\left(\Pi^{+}\right)$ if and only if it is the Fourier transform

$$
F(z)=\int_{0}^{\infty} f(t) e^{i z t} d t, \quad z \in \Pi^{+},
$$

of some function $f \in L_{\alpha+1}^{2}\left(\mathbb{R}^{+}\right)$, in which case $\|F\|_{\mathcal{A}_{\alpha}^{2}\left(\Pi^{+}\right)}=\|f\|_{L_{\alpha+1}^{2}\left(\mathbb{R}^{+}\right)}$.

The theorem says in particular that the unweighted Bergman space $\mathcal{A}^{2}\left(\Pi^{+}\right)$, where $\alpha=0$, is isometrically isomorphic to the space $L_{1}^{2}\left(\mathbb{R}^{+}\right)$with norm

$$
\|f\|_{L_{1}^{2}\left(\mathbb{R}^{+}\right)}^{2}=\pi \int_{0}^{\infty} \frac{|f(t)|^{2}}{t} d t
$$

This correspondence will allow us to view the shift operator as an operator on $L_{1}^{2}\left(\mathbb{R}^{+}\right)$. The shift operator $S$ is defined on $\mathcal{A}^{2}(\mathbb{D})$ by $S f(w)=w f(w)$ for $w \in \mathbb{D}$.

Theorem 2. The shift operator on the Bergman space $\mathcal{A}^{2}(\mathbb{D})$ is unitarily equivalent to the operator $I-2 T$ on $L_{1}^{2}\left(\mathbb{R}^{+}\right)$, where

$$
T f(t)=e^{-t} \int_{0}^{t} e^{s} f(s) d s, \quad f \in L_{1}^{2}\left(\mathbb{R}^{+}\right) .
$$

Note that $T$ is a convolution operator $T f=h * f$ with kernel

$$
h(t)= \begin{cases}e^{-t} & t>0 \\ 0 & t<0 .\end{cases}
$$

It can also be viewed as a Volterra operator combined with multiplication by $e^{-t}$.

A subspace $M$ of $\mathcal{A}^{2}(\mathbb{D})$ is said to be invariant if $S(M) \subset M$. Beurling [1] showed that the invariant subspaces of the shift operator on the Hardy space $\mathcal{H}^{2}(\mathbb{D})$ have the simple form $\varphi \mathcal{H}^{2}(\mathbb{D})$ where $\varphi$ is an inner function. On the other hand, the invariant subspaces of the Bergman space are much more complicated and have never been completely described (see for instance [3] for further discussion). Theorem 2 has the following immediate corollary.

Corollary. Under the unitary equivalence of Theorem 2, the invariant subspaces of the shift operator on $\mathcal{A}^{2}(\mathbb{D})$ correspond to the invariant subspaces of the operator $T$ on the space $L_{1}^{2}\left(\mathbb{R}^{+}\right)$.

The correspondence of the corollary will be illustrated by two examples. We will also obtain an analogue of the Paley-Wiener theorem for weighted Dirichlet spaces. 


\section{Proof of Theorem 1}

After discovering Theorem 1 we were informed that it is a "folk theorem", essentially known although there appears to be no proof in the literature. The proof we give here is not the shortest possible, but it reveals an interesting connection with Laguerre polynomials.

Let us show first that if $F$ has the form (1) for some function $f \in L_{\alpha+1}^{2}\left(\mathbb{R}^{+}\right)$, then $F \in \mathcal{A}_{\alpha}^{2}\left(\Pi^{+}\right)$and the two norms are equal. An application of Morera's theorem shows that $F$ is analytic in $\Pi^{+}$. Since the function $f(t) e^{-y t}$ belongs to $L^{2}\left(\mathbb{R}^{+}\right)$for each $y>0$, it follows from Plancherel's theorem that

$$
\begin{aligned}
\|F\|_{\mathcal{A}_{\alpha}^{2}\left(\Pi^{+}\right)}^{2} & =\int_{0}^{\infty} \int_{-\infty}^{\infty}|F(x+i y)|^{2} y^{\alpha} d x d y \\
& =\int_{0}^{\infty} \int_{-\infty}^{\infty}\left|\int_{0}^{\infty} f(t) e^{i t x} e^{-t y} d t\right|^{2} d x y^{\alpha} d y \\
& =\int_{0}^{\infty} \int_{0}^{\infty}\left|f(t) e^{-t y}\right|^{2} d t y^{\alpha} d y \\
& =\int_{0}^{\infty}|f(t)|^{2} \int_{0}^{\infty} e^{-2 t y} y^{\alpha} d y d t \\
& =2 \pi \int_{0}^{\infty}|f(t)|^{2} \frac{\Gamma(\alpha+1)}{(2 t)^{\alpha+1}} d t=\|f\|_{L_{\alpha+1}^{2}\left(\mathbb{R}^{+}\right)}^{2}
\end{aligned}
$$

A good reference for basic theory of Fourier transforms is Goldberg's book [4].

It remains to prove that the isometry $f \mapsto F$ maps onto the whole space $\mathcal{A}_{\alpha}^{2}\left(\Pi^{+}\right)$. In other words, we must show that every function $F \in \mathcal{A}_{\alpha}^{2}\left(\Pi^{+}\right)$has a representation of the form (1) for some function $f \in L_{\alpha+1}^{2}\left(\mathbb{R}^{+}\right)$. It will be sufficient to represent every element of an orthogonal basis in this manner.

Recall first that the weighted Bergman space $\mathcal{A}_{\alpha}^{2}(\mathbb{D})$ of the unit disk $\mathbb{D}$ consists of all analytic functions $g$ for which the integral

$$
\|g\|_{\mathcal{A}_{\alpha}^{2}(\mathbb{D})}^{2}=\int_{\mathbb{D}}|g(w)|^{2}\left(1-|w|^{2}\right)^{\alpha} d u d v, \quad w=u+i v,
$$

is finite. A change of variables shows that $g \in \mathcal{A}_{\alpha}^{2}(\mathbb{D})$ if and only if

$$
G(z)=g\left(\frac{z-i}{z+i}\right) \frac{2^{\alpha+1}}{(z+i)^{\alpha+2}}
$$

belongs to $\mathcal{A}_{\alpha}^{2}\left(\Pi^{+}\right)$. In fact, the linear map that takes $g \in \mathcal{A}_{\alpha}^{2}(\mathbb{D})$ onto $G \in \mathcal{A}_{\alpha}^{2}\left(\Pi^{+}\right)$ is an isometric isomorphism. Such an isometry preserves inner products and therefore carries any orthogonal basis of $\mathcal{A}_{\alpha}^{2}(\mathbb{D})$ to an orthogonal basis of $\mathcal{A}_{\alpha}^{2}\left(\Pi^{+}\right)$.

Since the polynomials are dense in $\mathcal{A}_{\alpha}^{2}(\mathbb{D})$ (see for instance [3]), the monomials $w^{n}$ with $n=0,1,2, \ldots$ form an orthogonal basis for $\mathcal{A}_{\alpha}^{2}(\mathbb{D})$. As a consequence, the functions

$$
G_{n}(z)=\left(\frac{z-i}{z+i}\right)^{n} \frac{2^{\alpha+1}}{(z+i)^{\alpha+2}}, \quad n=0,1,2, \ldots,
$$

form an orthogonal basis for $\mathcal{A}_{\alpha}^{2}\left(\Pi^{+}\right)$. The proof of Theorem 1 reduces to showing that each basis element $G_{n}$ is the image of some function $f_{n} \in L_{\alpha+1}^{2}\left(\mathbb{R}^{+}\right)$under 
the mapping defined by (1). For this purpose we need to compute the the Fourier transform

$$
\widehat{G}_{n}(t)=\frac{1}{2 \pi} \int_{-\infty}^{\infty} e^{-i t x} G_{n}(x) d x=\frac{1}{2 \pi} \int_{-\infty}^{\infty} e^{-i t x}\left(\frac{x-i}{x+i}\right)^{n} \frac{2^{\alpha+1}}{(x+i)^{\alpha+2}} d x
$$

The calculation of $\widehat{G}_{n}(t)$ will require some labor. We state the result as a lemma.

Lemma 1. The Fourier transform of $G_{n}$ is

$$
\widehat{G}_{n}(t)= \begin{cases}\frac{n !(2 t)^{\alpha+1} e^{-t}}{i^{\alpha+2} \Gamma(n+\alpha+2)} L_{n}^{(\alpha+1)}(2 t), & t>0 \\ 0, & t<0\end{cases}
$$

where $L_{n}^{(\alpha)}(t)$ denotes the Laguerre polynomial of degree $n$ and index $\alpha$.

The proof of Lemma 1 is deferred to Section 5, where we will say more about Laguerre polynomials. It is clear from formula (3) that $\widehat{G}_{n} \in L_{\alpha+1}^{2}\left(\mathbb{R}^{+}\right)$, and it also belongs to $L^{1}(\mathbb{R})$. Therefore, inversion of the Fourier transform yields a representation

$$
G_{n}(z)=\int_{0}^{\infty} e^{i z t} \widehat{G}_{n}(t) d t, \quad z \in \Pi^{+},
$$

of the form (1). Thus Theorem 1 will be proved once Lemma 1 is established.

\section{Proof of Theorem 2}

The linear mapping from $f \in \mathcal{A}^{2}(\mathbb{D})$ to the function

$$
F(z)=f\left(\frac{z-i}{z+i}\right) \frac{2}{(z+i)^{2}}, \quad z \in \Pi^{+}
$$

is an isometry of $\mathcal{A}^{2}(\mathbb{D})$ onto $\mathcal{A}^{2}\left(\Pi^{+}\right)$that carries the function $S f(w)=w f(w)$ to

$$
\frac{z-i}{z+i} F(z)=\widetilde{S} F(z)
$$

This shows that the shift operator in $\mathcal{A}^{2}(\mathbb{D})$ is unitarily equivalent to the operator $\widetilde{S}$ of multiplication by $(z-i) /(z+i)$ in $\mathcal{A}^{2}\left(\Pi^{+}\right)$.

Now recall that the functions

$$
G_{n}(z)=\left(\frac{z-i}{z+i}\right)^{n} \frac{2}{(z+i)^{2}}, \quad n=0,1,2, \ldots
$$

form an orthogonal basis of $\mathcal{A}^{2}\left(\Pi^{+}\right)$, and observe that $\widetilde{S} G_{n}=G_{n+1}$. Therefore, the Fourier transform of $G_{n+1}$ has the form

$$
\widehat{G}_{n+1}(t)=\frac{1}{2 \pi} \int_{-\infty}^{\infty} e^{-i t x} \frac{x-i}{x+i} G_{n}(x) d x .
$$

Write

$$
\frac{x-i}{x+i}=1-\frac{2}{1-i x}
$$


and note that the function $h$ given by (2) has the form

$$
h(t)=\frac{1}{2 \pi} \int_{-\infty}^{\infty} e^{-i t x} \frac{1}{1-i x} d x
$$

since

$$
\int_{-\infty}^{\infty} e^{i x t} h(t) d t=\int_{0}^{\infty} e^{-(1-i x) t} d t=\frac{1}{1-i x}
$$

It follows that the Fourier transform of $\widetilde{S} G_{n}$ is

$$
\begin{aligned}
\widehat{G}_{n+1}(t) & =\widehat{G}_{n}(t)-\frac{2}{2 \pi} \int_{-\infty}^{\infty} e^{-i t x} \frac{1}{1-i x} G_{n}(x) d x \\
& =\widehat{G}_{n}(t)-2\left(h * \widehat{G}_{n}\right)(t),
\end{aligned}
$$

where

$$
\left(h * \widehat{G}_{n}\right)(t)=\int_{-\infty}^{\infty} h(t-s) \widehat{G}_{n}(s) d s=e^{-t} \int_{0}^{t} e^{s} \widehat{G}_{n}(s) d s .
$$

Here we have used the fact that the Fourier transform of a convolution is the product of Fourier transforms.

We have shown that the multiplication operator $\widetilde{S}$, acting on a basis element $G_{n}$ of $\mathcal{A}^{2}\left(\Pi^{+}\right)$, is unitarily equivalent under the Fourier transform to the operator $I-2 T$ acting on $\widehat{G}_{n} \in L_{1}^{2}\left(\mathbb{R}^{+}\right)$. But by Theorem 1 , the Fourier transform is an isometric isomorphism from $\mathcal{A}^{2}\left(\Pi^{+}\right)$onto the space $L_{1}^{2}\left(\mathbb{R}^{+}\right)$, so this unitary equivalence extends to the full spaces. This completes the proof of Theorem 2.

\section{InVARIANT SUBSPACES OF THE BERGMAN SHIFT}

By the corollary to Theorem 2, the invariant subspaces of the shift operator on $\mathcal{A}^{2}(\mathbb{D})$ correspond, under the specified unitary equivalence, to the invariant subspaces of the operator $T$ in the space $L_{1}^{2}\left(\mathbb{R}^{+}\right)$. We now give two explicit examples of this correspondence.

Example 1. For any number $a>0$, the closed subspace of $L_{1}^{2}\left(\mathbb{R}^{+}\right)$consisting of functions that vanish on $[0, a]$ is invariant under $T$. It is not hard to see that it corresponds to the subspace

$$
M_{a}=\exp \left\{-a \frac{1+w}{1-w}\right\} \mathcal{A}^{2}(\mathbb{D})
$$

generated in the Bergman space by an atomic singular inner function. In particular, this confirms the known fact that $M_{a}$ is a proper invariant subspace of $\mathcal{A}^{2}(\mathbb{D})$. It also demonstrates the strict inclusion $M_{b} \subset M_{a}$ for $0<a<b$.

Example 2. Let $N_{\omega}$ be the subspace of functions in $\mathcal{A}^{2}(\mathbb{D})$ that vanish at a specified point $\omega \in \mathbb{D}$. Then $f \in N_{\omega}$ if and only if $\left\langle f, k_{\omega}\right\rangle=0$, where

$$
k_{\omega}(w)=\frac{1}{(1-\bar{\omega} w)^{2}}, \quad w \in \mathbb{D},
$$


is the reproducing kernel at $\omega$ for the space $\mathcal{A}^{2}(\mathbb{D})$. The isometric isomorphism (4) from $\mathcal{A}^{2}(\mathbb{D})$ onto $\mathcal{A}^{2}\left(\Pi^{+}\right)$is found to map the function $k_{\omega}$ to

$$
K_{\omega}(z)=\frac{2}{((z+i)-\bar{\omega}(z-i))^{2}}, \quad z \in \Pi^{+}
$$

and $N_{\omega}$ corresponds to the subspace of functions $F \in \mathcal{A}^{2}\left(\Pi^{+}\right)$with $\left\langle F, K_{\omega}\right\rangle=0$. A calculation shows that the Fourier transform

$$
\widehat{K}_{\omega}(t)=\frac{1}{2 \pi} \int_{-\infty}^{\infty} e^{-i t x} K_{\omega}(x) d x
$$

has the form $\widehat{K}_{\omega}(t)=t f_{\omega}(t)$, where

$$
f_{\omega}(t)=\frac{4 \pi}{(1-\bar{\omega})^{2}} \exp \left\{\frac{\bar{\omega}+1}{\bar{\omega}-1} t\right\} \quad \text { for } t>0
$$

and $f_{\omega}(t)=0$ for $t<0$. To see this, write $\lambda=(\bar{\omega}+1) /(\bar{\omega}-1)$ and observe that $\operatorname{Re}\{\lambda\}<0$ since $|\omega|<1$, so that

$$
F_{\omega}(x)=\int_{-\infty}^{\infty} e^{i x t} f_{\omega}(t) d t=\frac{2}{(1-\bar{\omega})^{2}} \int_{0}^{\infty} e^{(\lambda+i x) t} d t=\frac{2}{(1-\bar{\omega})^{2}} \frac{1}{\lambda+i x}
$$

has Fourier transform $\widehat{F}_{\omega}(t)=f_{\omega}(t)$. But $F_{\omega}^{\prime}(x)=i K_{\omega}(x)$ and $\widehat{F_{\omega}^{\prime}}(t)=i t \widehat{F}_{\omega}(t)$, so it follows that $\widehat{K}_{\omega}(t)=t f_{\omega}(t)$, as claimed.

Under the isometric isomorphism (4), the subspace $N_{\omega}$ in $\mathcal{A}^{2}(\mathbb{D})$ was seen to correspond to the orthocomplement $\left\langle K_{\omega}\right\rangle^{\perp}$ of the subspace $\left\langle K_{\omega}\right\rangle$ generated in $\mathcal{A}^{2}\left(\Pi^{+}\right)$ by the function $K_{\omega}$. But by Theorem 1 the space $\mathcal{A}^{2}\left(\Pi^{+}\right)$is isometrically isomorphic under the Fourier transform to the weighted space $L_{1}^{2}\left(\mathbb{R}^{+}\right)$, with inner product

$$
\langle f, g\rangle=\pi \int_{0}^{\infty} f(t) \overline{g(t)} \frac{1}{t} d t .
$$

In particular, the subspace $\left\langle K_{\omega}\right\rangle^{\perp}$ of $\mathcal{A}^{2}\left(\Pi^{+}\right)$corresponds to the subspace $\left\langle\widehat{K}_{\omega}\right\rangle^{\perp}$ of $L_{1}^{2}\left(\mathbb{R}^{+}\right)$under the Fourier transform. Since $\widehat{K}_{\omega}(t)=t f_{\omega}(t)$, we see that $f \in\left\langle\widehat{K}_{\omega}\right\rangle^{\perp}$ if and only if

$$
\int_{0}^{\infty} f(t) \exp \left\{\frac{\omega+1}{\omega-1} t\right\} d t=0 .
$$

This subspace $\left\langle\widehat{K}_{\omega}\right\rangle^{\perp}$ of $L_{1}^{2}\left(\mathbb{R}^{+}\right)$corresponds to the invariant subspace $N_{\omega}$ of the Bergman shift and is invariant under the operator $T$ defined in Theorem 2.

More generally, for any simple zero-set $\Omega$, the invariant subspace of functions in $\mathcal{A}^{2}(\mathbb{D})$ that vanish on $\Omega$ corresponds to the intersection of the subspaces $\left\langle\widehat{K}_{\omega}\right\rangle^{\perp}$ for all $\omega \in \Omega$.

\section{LAGUERRE POLYNOMIALS}

We now turn to a proof of Lemma 1, which evaluates the Fourier transforms of basis elements $G_{n}$ in terms of Laguerre polynomials. We begin by recording some facts about Laguerre polynomials. 
The Laguerre polynomials $L_{n}^{(\alpha)}(t)$ arise from the generating relation

$$
\frac{1}{(1-z)^{\alpha+1}} \exp \left\{-\frac{t z}{1-z}\right\}=\sum_{n=0}^{\infty} L_{n}^{(\alpha)}(t) z^{n} .
$$

For $\alpha>-1$ they have the orthogonality property

$$
\int_{0}^{\infty} e^{-t} t^{\alpha} L_{n}^{(\alpha)}(t) L_{m}^{(\alpha)}(t) d x=\Gamma(\alpha+1)\left(\begin{array}{c}
n+\alpha \\
n
\end{array}\right) \delta_{m n}, \quad n, m=0,1,2, \ldots
$$

They also have the explicit representation

$$
L_{n}^{(\alpha)}(t)=\sum_{j=0}^{n}\left(\begin{array}{c}
n+\alpha \\
n-j
\end{array}\right) \frac{(-t)^{j}}{j !}=\frac{\Gamma(n+\alpha+1)}{n !} \sum_{j=0}^{n}\left(\begin{array}{l}
n \\
j
\end{array}\right) \frac{(-t)^{j}}{\Gamma(\alpha+j+1)} .
$$

Further information may be found in the books by Szegö [9] or Rainville [7].

Although Fourier transforms of the Laguerre functions can be deduced from available formulas for their Laplace transforms, we carry out a proof of Lemma 1 for the sake of completeness. We will need another lemma.

Lemma 2. For $\beta>-1$ and $x \in \mathbb{R}$,

$$
\int_{0}^{\infty} t^{\beta} e^{-t} e^{i x t} d t=\frac{\Gamma(\beta+1)}{(1-i x)^{\beta+1}} .
$$

Lemma 2 follows from a simple property of the gamma function. The proof is omitted.

Proof of Lemma 1. By the identity

$$
\frac{x-i}{x+i}=1-\frac{2 i}{x+i}=1-\frac{2}{1-i x}
$$

and the binomial formula, we have

$$
\widehat{G}_{n}(t)=\frac{1}{4 \pi i^{\alpha+1}} \sum_{j=0}^{n}\left(\begin{array}{c}
n \\
j
\end{array}\right)(-1)^{j} \int_{-\infty}^{\infty} e^{-i t x}\left(\frac{2}{1-i x}\right)^{j+\alpha+2} d x .
$$

But inversion of the Fourier transform of Lemma 2 shows that

$$
\frac{1}{2 \pi} \int_{-\infty}^{\infty} e^{-i t x}\left(\frac{2}{1-i x}\right)^{j+\alpha+2} d x= \begin{cases}\frac{2^{j+\alpha+2}}{\Gamma(j+\alpha+2)} t^{j+\alpha+1} e^{-t}, & t>0 \\ 0, & t<0 .\end{cases}
$$

This gives $\widehat{G}_{n}(t)=0$ for $t<0$. For $t>0$ it gives

$$
\begin{aligned}
i^{\alpha+1} \widehat{G}_{n}(t) & =(2 t)^{\alpha+1} e^{-t} \sum_{j=0}^{n}\left(\begin{array}{c}
n \\
j
\end{array}\right) \frac{(-2 t)^{j}}{\Gamma(j+\alpha+2)} \\
& =\frac{n !}{\Gamma(n+\alpha+2)}(2 t)^{\alpha+1} e^{-t} L_{n}^{(\alpha+1)}(2 t),
\end{aligned}
$$

by comparison with the explicit representation (7) of the Laguerre polynomials. This completes the proof of Lemma 1.

We remark that the same method can be applied to prove the classical PaleyWiener theorem for the Hardy space of the upper half-plane. 


\section{Weighted Dirichlet SPACES}

As a direct corollary of Theorem 1, we can now formulate a Paley-Wiener theorem for weighted Dirichlet spaces of the upper half-plane. For $\alpha>-1$, a function $F$ analytic in $\Pi^{+}$is said to belong to the weighted Dirichlet space $\mathcal{D}_{\alpha}\left(\Pi^{+}\right)$if its derivative $F^{\prime}$ is in the weighted Bergman space $\mathcal{A}_{\alpha}^{2}\left(\Pi^{+}\right)$. Upon identifying functions that differ by a constant, the space $\mathcal{D}_{\alpha}\left(\Pi^{+}\right)$becomes a Hilbert space with norm

$$
\|F\|_{\mathcal{D}_{\alpha}\left(\Pi^{+}\right)}=\left\{\int_{\Pi^{+}}\left|F^{\prime}(x+i y)\right|^{2} y^{\alpha} d x d y\right\}^{1 / 2} .
$$

For $\alpha=0$, it is not hard to see that the space $\mathcal{D}_{0}\left(\Pi^{+}\right)=\mathcal{D}\left(\Pi^{+}\right)$is isometrically isomorphic to the Dirichlet space $\mathcal{D}(\mathbb{D})$ on the unit disc modulo constant functions. In fact, the composition operator $C_{\sigma}$ induced by $\sigma(z)=(z-i) /(z+i), z \in \Pi^{+}$, is an isometric isomorphism from $\mathcal{D}(\mathbb{D})$ onto $\mathcal{D}\left(\Pi^{+}\right)$.

Here is the Paley-Wiener theorem for weighted Dirichlet spaces.

Theorem 3. For each $\alpha>-1$, a function $F$ analytic in $\Pi^{+}$belongs to the space $\mathcal{D}_{\alpha}\left(\Pi^{+}\right)$if and only if it has the form

$$
F(z)=\int_{0}^{\infty} e^{i z t} f(t) d t, \quad z \in \Pi^{+},
$$

up to an additive constant, where $f$ is a measurable function on $\mathbb{R}^{+}$with

$$
\int_{0}^{\infty}|f(t)|^{2} t^{1-\alpha} d t<\infty
$$

Moreover,

$$
\|F\|_{\mathcal{D}_{\alpha}\left(\Pi^{+}\right)}^{2}=\frac{\Gamma(\alpha+1)}{2^{\alpha}} \int_{0}^{\infty}|f(t)|^{2} t^{1-\alpha} d t .
$$

The unweighted case of the theorem $(\alpha=0)$ was previously proved by Higdon [5], using the fact that $C_{\sigma}$ is a unitary operator from $\mathcal{D}(\mathbb{D})$ onto $\mathcal{D}\left(\Pi_{+}\right)$, together with the classical Paley-Wiener Theorem for $\mathcal{H}^{2}\left(\Pi^{+}\right)$.

Proof of Theorem 3. If $F \in \mathcal{D}_{\alpha}\left(\Pi^{+}\right)$, then $F^{\prime} \in \mathcal{A}_{\alpha}^{2}\left(\Pi^{+}\right)$and therefore, Theorem 1 provides us with a function $g \in L_{\alpha+1}^{2}\left(\mathbb{R}^{+}\right)$such that

$$
F^{\prime}(z)=\int_{0}^{\infty} e^{i z t} g(t) d t, \quad z \in \Pi^{+} .
$$

It follows that, up to an additive constant,

$$
F(z)=\int_{0}^{\infty} e^{i z t} f(t) d t, \quad z \in \Pi^{+},
$$

where itf $f(t)=g(t)$. The remaining assertions follow directly from Theorem 1 .

It may be remarked that this function $F$, represented by a Fourier transform, is the unique member of its equivalence class with the property that $F(x+i y) \rightarrow 0$ as $y \rightarrow \infty$. 


\section{ACKNOWLEDGMENTS}

This work was partially carried out while the second author was visiting the University of Michigan in the Fall Term of 2004, with a grant from Secretaría de Estado de Educación y Universidades. She thanks the Mathematics Department at the University of Michigan for its hospitality.

\section{REFERENCES}

[1] A. Beurling, On two problems concerning linear transformations in Hilbert space, Acta Math. 81 (1948), 239-255.

[2] P. L. Duren, Theory of $H^{p}$ Spaces, Academic Press, New York, 1970; reprinted with supplement by Dover Publications, Mineola, N. Y., 2000.

[3] P. Duren and A. Schuster, Bergman Spaces, American Mathematical Society, Providence, R I., 2004.

[4] R. R. Goldberg, Fourier Transforms, Cambridge University Press, Cambridge, U. K., 1961.

[5] W. M. Higdon, The spectra of composition operators from linear fractional maps acting upon the Dirichlet space, J. Funct. Anal. 220 (2005), 55-75.

[6] R. E. A. C. Paley and N. Wiener, Fourier Transforms in the Complex Domain, American Mathematical Society, Providence, R. I., 1934.

[7] E. D. Rainville, Special Functions, Chelsea Publ. Company, New York, 1971.

[8] W. Rudin, Real and Complex Analysis, Third Ed., McGraw-Hill, New York, 1987.

[9] G. Szegö, Orthogonal Polynomials, Fourth Ed., American Mathematical Society, Providence, R. I., 1975.

Department of Mathematics, University of Michigan, Ann Arbor, Michigan 481091109, U.S.A.

E-mail address: duren@umich.edu

Departamento de Matemáticas, Universidad de Zaragoza, Plaza San Francisco S/N, 50009 ZARAGOZA, SPAIN

E-mail address: eva@unizar.es

Departamento de Análisis Matemático, Facultad de Matemáticas, Avenida Reina Mercedes, Apartado 1160, Sevilla 41080, SPAiN

E-mail address: amontes@us.es 